\title{
Implantación de alojamientos en el medio rural y freno a la despoblación: realidad o ficción. El caso de Extremadura (España) ${ }^{1}$
}

\author{
Accommodation businesses as a meanss against \\ population decline: reality or illusion? Extremadura \\ (Spain) as a case-study
}

\author{
José Manuel Sánchez Martín² (), Juan Ignacio Rengifo Gallego ${ }^{3}$ (1) \\ y Rocío Blas Morato ${ }^{4}$ [i]
}

\begin{abstract}
RESUMEN
Los municipios rurales se enfrentan a una despoblación paulatina que, unida a otros problemas derivados de la misma, ha favorecido la implantación de numerosas políticas de desarrollo rural para combatirla. Desde diferentes administraciones se ha apostado por el fomento del turismo, si bien, en el caso de Extremadura, no se ha alcanzado el objetivo marcado. Los municipios con una población inferior a 10.000 habitantes, sobre los que se ha asentado una importante planta de alojamientos, continúan perdiendo efectivos pese a disponer de importantes recursos turísticos. Ello implica el fracaso de las políticas turísticas, encaminadas a incrementar la capacidad de hospedaje frente a la necesidad de generar productos partiendo de las preferencias que manifiesta la demanda. Con ello se constata que para frenar la despoblación son necesarias políticas de mayor calado que la mera implantación de alojamientos o la oferta de productos turísticos poco realistas y de escasa proyección en el territorio analizado.
\end{abstract}

Palabras clave: Alojamiento, desarrollo rural, despoblación, Extremadura, turismo rural.

\begin{abstract}
Rural towns and villages are facing a continuous population decline which, together with other related problems, has called for different development policies in order to counteract it. Different administrations have chosen to develop the tourist sector-even though in the case of Extremadura these projects did not reach the goals they sought to achieve. Towns under 10,000 inhabitants, where a relatively large number of tourist accommodation businesses have been established, are still losing population despite their potential and tourist-related resources. This implies that the policies to expand tourism have failed, especially because they have focused on enlarging accommodation capacity instead of generating products that meet the demands of customers. This attests to the notion that different policies are necessary in order to stop population decline-rather than simply establishing accommodation businesses or offering unrealistic tourist products that are unlikely to have a noticeable impact on the area object of study.
\end{abstract}

Key words: Accommodation, rural development, population decline, Extremadura, rural tourism. 
Los entornos rurales se han definido de formas diversas, bajo distintas percepciones y marcos geográficos. Son numerosos los organismos internacionales que han tratado la delimitación de este tipo de áreas, como la OCDE, Eurostat, etc., si bien, a efectos de este artículo, se parte de la conceptualización que realiza en España la Ley de Desarrollo Sostenible del Medio Rural. Según dicho precepto legislativo "se entiende por:

a) Medio rural: el espacio geográfico formado por la agregación de municipios o entidades locales menores definido por las administraciones competentes que posean una población inferior a 30.000 habitantes y una densidad inferior a los 100 habitantes por km².

b) Zona rural: ámbito de aplicación de las medidas derivadas del Programa de Desarrollo Rural Sostenible regulado por esta Ley, de amplitud comarcal o subprovincial, delimitado y calificado por la Comunidad Autónoma competente.

c) Municipio rural de pequeño tamaño: el que posea una población residente inferior a los 5.000 habitantes y esté integrado en el medio rural" (Ley 45/2007 para el desarrollo sostenible del medio rural, 2007: 51.342).

Los municipios rurales de pequeño tamaño y las zonas que configuran se enfrentan a numerosos problemas derivados de la despoblación secular a que se han visto sometidos, al fuerte envejecimiento de la población residente, a la progresiva masculinización y a la debilidad económica de los sectores productivos, con una fuerte representatividad del sector primario, básicamente agricultura y ganadería, así como a la carencia de infraestructuras y servicios de calidad.

La despoblación no es un fenómeno reciente, sino que se remonta a los años del desarrollismo frenético de los años 60, fruto de la industrialización de determinadas áreas de España (País Vasco, Cataluña o Madrid). Sus causas y consecuencias han sido estudiadas de forma profusa en la literatura especializada de épocas muy distantes (Bringas, 1963; Slomp, 2004); si bien algunos autores refuerzan la necesidad de diferenciar la despoblación dependiendo de su génesis. Así, distinguen entre la originada por el exceso de defunciones sobre nacimientos, lo que a su vez repercute en mayor envejecimiento de la población; la producida por la emigración hacia otros destinos, que a la postre degenera en mayor envejecimiento; y, por último, la desertización, entendida desde el punto de vista demográfico, producida cuando un núcleo ha perdido a todos sus habitantes (López, Abellán \& Godenau, 2009).

A pesar de que el despoblamiento rural ha sido una constante en España desde los años 60 , cabe mencionar que a partir de los 80 se observan cambios de tendencia, pues las grandes ciudades experimentan menor crecimiento porcentual de la población que las zonas periféricas (Pozo \& García, 2009), como ha sucedido en Madrid. No obstante, esta misma propensión se ha detectado también en entornos con menor peso demográfico como sucede en la periferia de las principales ciudades de Castilla y León, Castilla - La Mancha o Extremadura.

Para hacer frente a la despoblación de los entornos rurales, se aprueba la Ley 45/2007, de 13 de diciembre, para el desarrollo sostenible del medio rural, en cuyo preámbulo ya se destaca la trascendencia de estos entornos en España: 
"La importancia actual del medio rural en España, que integra al 20 por ciento de la población, que se elevaría hasta el 35 por ciento si se incluyen las zonas periurbanas y afecta al 90 por ciento del territorio, $y$ el hecho de que en este inmenso territorio rural se encuentran la totalidad de nuestros recursos naturales y una parte significativa de nuestro patrimonio cultural, así como las nuevas tendencias observadas en la localización de la actividad económica y residencial, confieren a este medio una relevancia mayor de la concedida en nuestra historia reciente" (BOE, 2007: 51.339).

Asimismo, el artículo 20 de dicha Ley, propone diferentes medidas para incentivar la diversificación económica en el medio rural. Entre ellas, el punto e recoge:

"Fomentar el turismo rural, en particular a través de una adecuada ordenación de la oferta y la mejora de la demanda turística, con una atención preferente al fomento de un turismo sostenible en las zonas rurales prioritarias, y al agroturismo o turismo ligado a la actividad agraria" (BOE, 2007: 51.344).

La apuesta por la defensa del medio rural que se hace desde diferentes instancias se materializa en el Real Decreto 1336/2011, de 3 de octubre, por el que se regula el contrato territorial como instrumento para promover el desarrollo sostenible del medio rural. El mismo apunta que "los contratos territoriales se conciben como un instrumento de apoyo a las políticas de desarrollo rural sostenible, con la intención de orientar e incentivar las actividades agrarias, entendidas en un sentido lo más amplio posible, hacia la multifuncionalidad y la generación de externalidades positivas que contribuyan eficazmente a mejorar los aspectos económicos, sociales y ambientales que configuran la sostenibilidad del medio rural, todo ello bajo la aplicación de un enfoque territorial" (BOE, 2011: 104.199).

No obstante, el parco avance de la economía rural respecto a la urbana, más centrada en la industria y los servicios, ha provocado notables corrientes migratorias hacia las ciudades en las que se demandaba numeroso empleo, en muchos casos mejor remunerado y sin estar sometido a las tensiones y vicisitudes del empleo agrario o ganadero. Ello sentó las bases del éxodo rural en España durante la década de los 60 , tal como se ha puesto de relieve en la literatura (Erdozáin \& Mikalerena, 1996; Silvestre, 2002; Atance et al., 2010).

El desarrollo económico actúa como desencadenante de los problemas demográficos, cuya preocupación centra numerosos debates en las administraciones públicas de distinto nivel. El trasfondo de los mismos es la aspiración generalizada de mejorar la calidad de vida, que infundía a los espacios urbanos la idea de que eran mejores en lo cultural e incluso en lo moral (Entrena, 2012; Paniagua, 2017). Afortunadamente, esa idea denostada del mundo rural ha ido cambiando y, durante las tres últimas décadas, se han producido procesos de revalorización y de revitalización socioeconómica y demográfica en numerosos espacios rurales, cuya cultura y formas de vida tienden a ser apreciadas en contraste con la visión peyorativa que predominaba durante las décadas de máxima vigencia del desarrollismo (García, 1997). Sin embargo, la pérdida de población continúa en una parte considerable de los núcleos de menor tamaño y afecta a numerosas áreas interiores de España (Baraja, 2003; López, 2009; Bustos, 2018).

Extremadura, zona elegida para el estudio, no ha sido ajena a la pérdida de población, cuyo destino final eran y siguen siendo los grandes polos de desarrollo industrial y, consecuentemente, las grandes ciudades del país. En esta comunidad autónoma, el descenso demográfico se 
constata con los datos de los diferentes censos oficiales publicados por el Instituto Nacional de Estadística (INE), que muestran que entre 1960 y 2001 se ha perdido más del $23 \%$ de la población absoluta, con ligeras recuperaciones de la misma en 2011, como consecuencia del retorno producido por la crisis económica, para volver a descender a medida que mejoraba la situación, según se constata en 2017. Por el contrario, el escenario que muestra el conjunto de España es muy diferente, pues se aprecia un importante crecimiento que supera el 52\% entre 1960 y 2017.

Esta situación contrapuesta entre una comunidad autónoma periférica y el conjunto del país se debe a los flujos migratorios, generalizados a partir de 1960, entre otras causas (Sánchez, 2011), época en la que muchos núcleos rurales llegaron a perder un porcentaje notable de su población joven, con el consiguiente riesgo que supone para la viabilidad demográfica de un territorio. Décadas después han comenzado a surgir importantes problemas en el medio rural que han desencadenado una fuerte crisis demográfica, fruto de la despoblación, la masculinización social y el envejecimiento, llegando a una situación casi insostenible en los núcleos de menor tamaño (Camarero \& Sampedro, 2008).

\section{Cuadro No 1}

Evolución de la población

\begin{tabular}{|r|r|r|r|r|}
\hline & \multicolumn{1}{|c|}{ España } & \multicolumn{1}{c|}{$\mathbf{N}^{\mathbf{0} \text { indice }}$} & \multicolumn{1}{c|}{ Extremadura } & \multicolumn{1}{c|}{$\mathbf{N}^{\mathbf{0}}$ índice } \\
\hline 1950 & 27.976 .755 & 100,0 & 1.364 .857 & 100,0 \\
\hline 1960 & 30.528 .539 & 109,1 & 1.379 .072 & 101,0 \\
\hline 1970 & 34.040 .989 & 121,7 & 1.169 .354 & 85,7 \\
\hline 1981 & 37.683 .362 & 134,7 & 1.064 .976 & 77,8 \\
\hline 1991 & 38.872 .268 & 138,9 & 1.061 .852 & 77,8 \\
\hline 2001 & 40.847 .371 & 146,0 & 1.058 .503 & 77,6 \\
\hline 2011 & 46.667 .175 & 166,8 & 1.102 .300 & 80,8 \\
\hline 2017 & 46.528 .966 & 166,3 & 1.077 .715 & 79,0 \\
\hline
\end{tabular}

Fuente: Instituto Nacional de Estadística (INE).

Ante el panorama desolador que se observa en muchas áreas rurales que ostentan los mayores índices de envejecimiento y la renta per cápita más baja, el turismo rural comenzó a verse como un factor diversificador e incrementador de las rentas agrarias (García, 1996), muy escasas en buena parte de los casos y, además, sometidas a las fluctuaciones vinculadas a un entorno metorológico cambiante, típico de áreas mediterráneas (Sánchez, 1995). Esta idea generalizada de que el potencial incremento de rentas conseguiría fijar a la población se ha ido extendiendo por todas las áreas interiores, obviando a veces la necesidad de plantear otras cuestiones de base como la automovilidad y las oportunidades de empleo que pueden surgir con ella (Camarero et, 2009); la necesidad de disponer de servicios sanitarios, educativos, etc (Escribano, 2012). Surge así la necesidad de plantearse la vinculación que existe entre la población y el turismo rural en áreas rurales, caracterizadas por su baja densidad demográfica (Jurado \& Pazos, 2016).

Se han realizado numerosas iniciativas públicas en el medio rural, conducentes en la mayor parte de los casos al fomento del turismo como un instrumento de desarrollo sostenible, fraca- 
sando en numerosas ocasiones según la opinión de varios expertos (Cànoves, Villarino \& Herrera, 2006; Sharpley \& Roberts, 2004, Yubero \& García, 2019).

Los motivos por los que buena parte de los proyectos no alcanzan el éxito son muy variados, tal como recoge la literatura, aunque existe cierto consenso cuando se atribuye a una evaluación errónea de las posibilidades de éxito (Tirado \& Hernández, 2017, 2018), a la falta de planificación sobre los lugares idóneos, fruto del desconocimiento del potencial turístico que posee el territorio (Sánchez, Sánchez \& Rengifo, 2017), e incluso a la presunción de que los recursos existentes en el territorio pueden ser capaces de atraer a la demanda (Prats, 2011), entre otros.

Resulta un hecho evidente que el medio rural está sometido a importantes tensiones, tanto demográficas como económicas, por lo que se ha tratado de vincularlo a una modalidad turística que tampoco está exenta de problemas, la rural (Sánchez \& Rengifo, 2019). En este sentido, se debate sobre el papel que puede desempeñar el turismo en estos territorios, atribuyéndole el papel de salvador del medio rural, en muchos casos careciendo de datos científicos. De ese modo, se observa un interés creciente por vincular aspectos demográficos y patrimoniales, a la vez que existe cierta predilección por desarrollar estos espacios de forma endógena y sostenible partiendo de la riqueza patrimonial, exuberante en muchos casos, y poniéndolos en valor para el aprovechamiento del turismo desde el prisma de la sostenibilidad (Martín \& Martín, 2014; Martín \& Martín, 2016), si bien, también se analiza el éxito de las políticas de desarrollo rural en diferentes facetas (Zasada et al., 2018). Obviamente la apuesta por el desarrollo sostenible y armónico de los territorios rurales pasa por la necesidad de integrar el resto de sectores productivos. Por ello, a lo largo de las dos últimas décadas se han generalizado los intentos por desarrollar el turismo rural en diferentes espacios, cuya evolución ha sido tratada profusamente en la literatura (Cánoves et al., 2004; Barke, 2004), así como sus implicaciones económicas (Mesa, 2000) e incluso proponiendo un marco teórico para el desarrollo del turismo comunitario como alternativa (Vinasco, 2017).

Las zonas rurales se han convertido en uno de los ejes de actuación centrales de iniciativas comunitarias de desarrollo rural auspiciadas por la Unión Europea entre las que cabe destacar: PRODER (1996-1999), PRODER2 (2000-2006), LEADER I (1991-1994), LEADER II (1994-1999), LEADER+ (2000-2006) o FEADER (2007-2013 y 2014-2020).

Entre las diferentes partidas presupuestarias de estas iniciativas se encuentran las orientadas al desarrollo turístico, en concreto, encaminadas hacia el turismo rural (Jurado \& Pazos, 2016). Abarcan desde la ayuda a la conservación del medio rural, con especial atención a los atractivos turísticos, hasta la puesta en marcha de productos específicos, así como a la mejora de la planta de alojamientos. No obstante, y a pesar de la fuerte inversión destinada a los mismos, los entornos rurales han continuado perdiendo población, aunque no en todos los casos. De hecho, en la literatura se observan diferentes proyectos que muestran opciones variadas para conseguir desarrollar económicamente estos espacios (Moreno, Ruíz \& Espín, 2017), a la vez que reflejan la casuística tan diferente que caracteriza cada espacio. Por ello, se cree necesario realizar un estudio específico para cada lugar, ya que los condicionantes de cada territorio marcan diferencias notables.

Todo ello da lugar a que este artículo se plantee como principal hipótesis la corroboración de que la implantación de alojamientos de forma masiva y a veces descoordinada no ha sido capaz de frenar el avance de la despoblación en los núcleos rurales, para lo que se ha tomado como 
referencia espacial Extremadura (España), así como la variación de los efectivos demográficos y la planta de hospedaje en dos años diferentes, 2004 y 2017. La asunción de esta hipótesis se debe a que, en su génesis, el turismo rural tenía como objetivo fundamental el complemento de las rentas agrarias y, por extensión, la fijación de la población al medio rural. Obviamente, ello no cuestiona que sean precisas otras actuaciones encaminadas al mantenimiento de la población.

La comprobación de dicha hipótesis implica cumplir con los siguientes objetivos de investigación:

a) determinar la evolución reciente de la población y fijar el conjunto de municipios que cumplen con el criterio de "municipio rural pequeño" según la Ley 45/2007;

b) estudiar la evolución, en diferentes fases, de la oferta de alojamientos, aunque enmarcándolo en el contexto general de la comunidad;

c) comprobar si existen relaciones estadísticas entre ambas circunstancias; $y$

d) establecer relaciones espaciales y determinar si existen áreas o zonas específicas donde se ha conseguido fijar a la población o, por el contrario, las medidas no lo han conseguido.

Aparte de esta introducción, el artículo se estructura en otros 3 apartados. La metodología utilizada se trata en el apartado segundo, en la que se realiza una breve caracterización del área de estudio, un somero análisis de las fuentes de datos utilizadas y se expone el método y las técnicas utilizadas. Los resultados y su discusión ocupan el apartado central de la investigación, en la que se analizan y comparan los datos referidos a la población y a la planta de alojamientos, lo que da lugar a que se pueda aseverar que hay espacios, incluso dentro de la propia zona de estudio, en los que el desarrollo del turismo contribuye a fijar población, aunque, obviamente, depende también de otros condicionantes. Por último, existe otro apartado donde se sintetizan las principales conclusiones que se extraen de este estudio.

\section{Metodología}

\section{Zona de estudio}

Extremadura es una comunidad autónoma española que se caracteriza por su posición periférica y marginal respecto a los principales centros económicos de la península ibérica. Además, durante décadas, ha estado aislada por su carácter fronterizo con Portugal (Rengifo \& Sánchez, 2017). Dicha situación no ha mejorado de forma sustancial a raíz de la incorporación de España a la Unión Europea, pues el efecto "frontera" permanece vigente.

Si a ello se le añade el olvido secular de la Administración Central a la hora de realizar inversiones con la finalidad de mejorar la accesibilidad y otros servicios esenciales, hay que afirmar que esta situación no favorece el desarrollo económico y explica, a su vez, los actuales problemas sociales y demográficos que caracterizan a la región. En este sentido, cabe destacar que no ha sido hasta épocas recientes cuando se han producido mejoras ostensibles en la articulación del sistema de comunicaciones terrestres por carreteras, centrado sobre todo en la creación de autovías que favorecen las conexiones intra y supra regionales, como la A-5, que une Madrid con Lisboa (1990-1993); la A-66, que enlaza Gijón con Sevilla (2004-2008); la EXA1, que une Naval- 
moral de la Mata con Moraleja y la EXA2, que comunica Miajadas con las Vegas Altas (Sánchez; Rengifo \& Martín, 2018).

Figura No 1

Localización del área de estudio

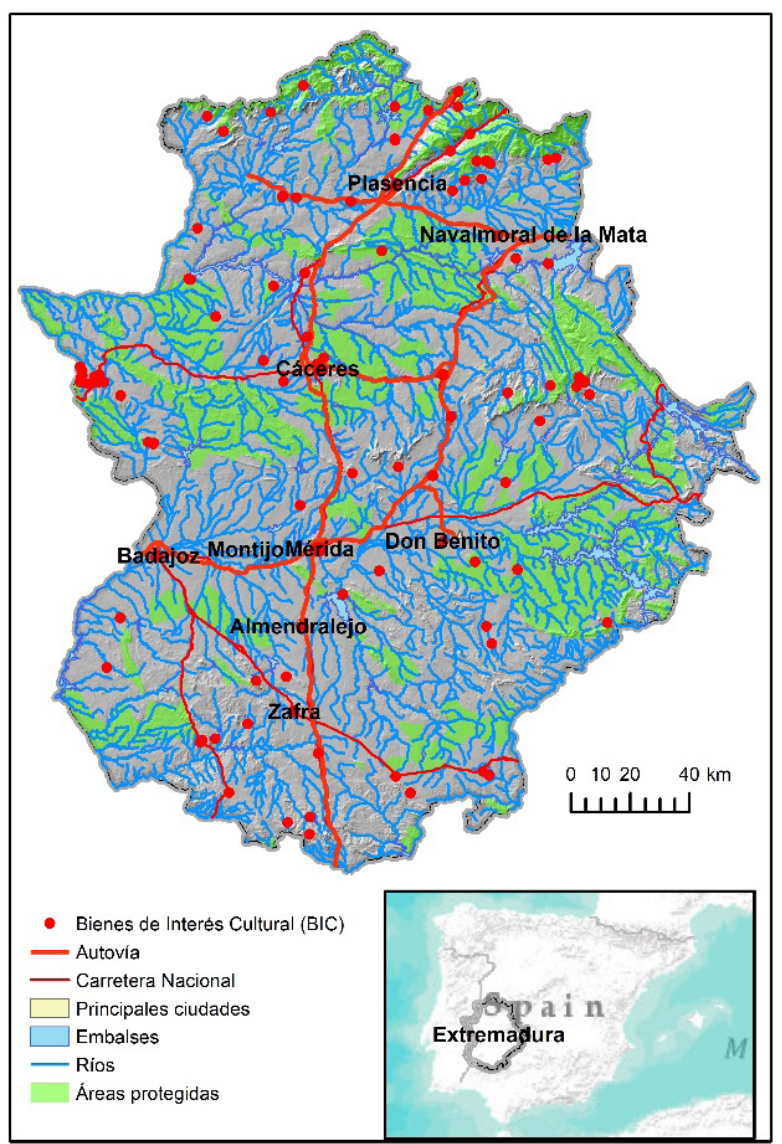

Fuente: Elaboración propia

Sin embargo, la conectividad por ferrocarril y por avión es muy limitada. En el primer caso por la presencia de una red obsoleta y carente de rutas viables a la vez que competitivas. En el segundo, porque, aunque el aeropuerto de Badajoz opera de forma intermitente desde 1990, no ha tenido continuidad temporal y las diferentes líneas que han operado en él desde su puesta en funcionamiento, han comunicado Badajoz con muy pocos destinos, haciéndolo a la postre poco competitivo (Sánchez \& Rengifo, 2017; Gurría, Sánchez \& Hernández, 2017). La restringida accesibilidad contrasta con la enorme riqueza cultural y natural que tiene buena parte del territorio, que cuenta con 34 Conjuntos Histórico-Artísticos y numerosas áreas protegidas mediante la Ley 9/2006, de 23 de diciembre (Rengifo \& Sánchez, 2016; Sánchez, Sánchez \& Rengifo, 2017). 


\section{Fuentes de información}

Para elaborar esta investigación se ha partido de la información proporcionada por varios organismos oficiales. En concreto, se han recopilado los datos ofrecidos por el Registro de Actividades Turísticas dependiente de la Dirección General de Turismo de la Junta de Extremadura; asimismo, se aportan datos publicados por el Instituto Nacional de Estadística (INE) y, por último, se recurre a la cartografía suministrada por el Instituto Geográfico Nacional (IGN).

La información que se ha conseguido recopilar es variada, si bien, en aras de la simplicidad, se sintetiza que el INE ha suministrado la información relativa a la población, tomando como referencia el padrón municipal de habitantes, tanto de 2004 como de 2017. En cambio, la Dirección General de Turismo de la Junta de Extremadura ha aportado toda la información relativa a las infraestructuras de alojamiento turístico, haciendo especial hincapié en los relativos al tipo de oferta, sus categorías y plazas, además de la ubicación. Por su parte, el IGN ha suministrado el soporte cartográfico que se incluye en la Base Topográfica Nacional a escala 1:100.000 en su versión de 2017. Dicha escala, pese a no contar con un excesivo detalle, proporciona la información necesaria para la realización de esta investigación.

La utilización conjunta de estas fuentes ha permitido generar una base de datos que contiene 60 variables referidas a población y a oferta de alojamientos para los años 2004 y 2017. Además de esta información cuantitativa, se dispone de una base cartográfica que posibilita añadir numerosos datos referidos a la localización, relieve, comunicaciones, etc.

Sendos tipos de información, la cuantitativa y la cartográfica, permiten la representación de las principales variables y, a la vez, la utilización de diferentes técnicas de análisis.

\section{Método y técnicas de análisis}

La sistemática de la investigación empleada se centra en la utilización combinada del método comparativo, de larga tradición en las Ciencias Sociales, basado en la comparación sistemática con el objetivo de verificar la hipótesis planteada, y el método inductivo. En este sentido, cabe señalar que la comparación es el criterio de la interpretación valorativa de los resultados empíricos (Gómez, 2014). Mientras tanto, la inducción muestra un razonamiento que va de lo particular a lo general, extrayendo conclusiones a partir de la observación sistemática y periódica de los hechos reales que ocurren en torno al fenómeno en estudio, con el propósito de descubrir las relaciones constantes derivadas del análisis y, con base en ellas, establecer hipótesis que, de comprobarse, adquieren el rango o categoría de leyes (Tamayo, 1990: 143).

De ese modo, se ha procedido a comparar la evolución que ha experimentado la población en los municipios extremeños entre 2004 y 2017, y se han categorizado en función del volumen de población residente en ellos durante los años analizados. La elección de esos años se ha debido a que en 2004 se había asentado plenamente el crecimiento del turismo rural en Extremadura, a la vez que las iniciativas europeas encaminadas al desarrollo rural habian adquirido madurez suficiente como para comenzar a dar resultados fehacientes. En cambio, el último año se ha tomado como base para determinar si la implantación de alojamientos rurales y, consecuentemente, el desarrollo del sector ha sido capaz de frenar la despoblación del mundo rural. 
Dado que se sigue una metodología comparativa e inductiva, se ha analizado la situación en los núcleos rurales, pero también en los urbanos, lo que a la postre permitirá determinar si existen grandes variaciones en el comportamiento existente entre ambos tipos de núcleo. A la vez se ha hecho lo propio con la oferta de alojamientos durante esos mismos años, diferenciando en este caso entre la situación conjunta de todos ellos y desagrupándola en las tres tipologías de alojamiento, lo que posibilitará confrontar el comportamiento detectado entre la modalidad de alojamiento rural, el hotelero y el extrahotelero. Para ello se consideran los establecimientos reconocidos por la Ley 2/2011, de 31 de enero, de desarrollo y modernización del turismo de Extremadura (DOE núm. 22 de 02 de febrero de 2011 y BOE núm. 42 de 18 de febrero de 2011).

Con ellos se ha procedido a generar una base de datos que, posteriormente, se ha tratado de forma estadística con SPSS v.21 y cartográfica con ArcGIS v.10.5. El software estadístico ha posibilitado extraer estadísticos descriptivos como la tabla de contingencia, utilizada para mostrar las características más significativas de cada grupo de municipios. Asimismo, se ha utilizado para determinar el coeficiente de correlación lineal entre las variables demográficas y de oferta de hospedaje durante los años analizados. El software SIG ha permitido cartografiar las variables y realizar el oportuno análisis de distribución territorial, pues permite una buena radiografía del estado de la población y del poblamiento, entre otros aspectos, tanto para los municipios rurales como urbanos, como lo han referido numerosos autores (Roquer \& Blay, 2008; Jurado \& Pazos, 2016).

\section{Resultados y discusión}

El análisis comparativo sobre la evolución paralela seguida por la planta de alojamientos y la población en municipios agrupados según su tamaño muestra que, para el conjunto de Extremadura, sendas variables siguen tendencias contrarias. De hecho, a pesar de experimentar incrementos globales en dichos parámetros, cuando se recurre al análisis territorial desagregado por términos municipales en función del tamaño de la población residente, las plazas de alojamiento aumentan, si bien, la población disminuye en el grupo de municipios que tienen 10.000 habitantes o menos. A este respecto cabe advertir que el conjunto de núcleos donde residen menos de 500 habitantes muestra un crecimiento de población, pero éste se debe a que el número de núcleos que lo integran se ha incrementado de forma notable, al pasar de 95 a 115. Además, cabría advertir que durante el periodo de estudio (2004-2017) se ha observado una clara polarización de la población, ya que esta aumenta en los núcleos de mayor entidad demográfica y disminuye de forma acusada en los más pequeños. Por ello, se puede afirmar que, de forma genérica, en los núcleos de hasta 5.000 residentes predomina la pérdida de población, mientras que los que superan los 10.000 habitantes, experimentan un incremento de la población.

Esta situación contrasta con la evolución seguida por la capacidad que ofrece la planta de hospedaje, donde se observa un incremento en todos los grupos considerados. Además, se revela que los incrementos más significativos se producen en los conjuntos de municipios integrados en el grupo que cuenta con una población igual o inferior a 10.000 habitantes. 


\section{Cuadro $\mathrm{N}^{\circ} 2$}

Comparativa entre población y plazas de hospedaje en Extremadura

\begin{tabular}{|l|r|r|r|r|r|r|}
\hline $\begin{array}{l}\text { Tamaño de } \\
\text { población }\end{array}$ & $\begin{array}{c}\mathbf{N}^{\circ} \text { Munic. } \\
\mathbf{2 0 0 4} \mathbf{( 3 8 3 )}\end{array}$ & $\begin{array}{c}\mathbf{N}^{\mathbf{0}} \text { Munic. } \\
\mathbf{2 0 1 7} \mathbf{( 3 8 8 )}\end{array}$ & $\begin{array}{c}\text { Plazas en } \\
\text { aloj. 2004 }\end{array}$ & $\begin{array}{l}\text { Plazas en } \\
\text { aloj. 2017 }\end{array}$ & $\begin{array}{c}\text { Población } \\
\mathbf{2 0 0 4}\end{array}$ & $\begin{array}{c}\text { Población } \\
\mathbf{2 0 1 7}\end{array}$ \\
\hline Menos de 500 & 95 & 115 & 546 & 2.004 & 27.593 & 30.479 \\
\hline 500 a 1.000 & 99 & 95 & 2.988 & 5.790 & 70.839 & 63.446 \\
\hline 1.001 a 2.000 & 85 & 83 & 3.803 & 5.459 & 121.207 & 111.962 \\
\hline 2.001 a 5.000 & 65 & 58 & 8.691 & 12.108 & 190.205 & 182.263 \\
\hline 5.001 a 10.000 & 25 & 24 & 2.924 & 4.012 & 166.003 & 161.226 \\
\hline 10.001 a 20.000 & 7 & 6 & 1.425 & 1.978 & 94.638 & 95.295 \\
\hline 20.001 a 50.000 & 4 & 4 & 2.101 & 2.505 & 125.221 & 138.173 \\
\hline Más de 50.000 & 3 & 3 & 5.492 & 7.433 & 279.580 & 304.934 \\
\hline TOTAL & 383 & 388 & 27.970 & 41.289 & 1.075 .286 & 1.087 .778 \\
\hline
\end{tabular}

Fuente: Elaboración propia a partir de INE y Junta de Extremadura

No obstante, la situación descrita se refiere al conjunto de municipios de Extremadura, aunque sea articulados a través de grupos determinados por su población, sea cual fuere su condición respecto al nivel de desarrollo turístico. Por ello, es preciso realizar una discriminación entre los que poseen alojamientos y los que carecen de ellos durante el año 2017, con el objetivo de comprobar si las tendencias demográficas son similares $y$, sobre todo, si la apuesta por el turismo ha sido capaz de cumplir con uno de los requerimientos básicos del desarrollo rural, fijar la población.

Cuadro No 3

Población y plazas de hospedaje en municipios que disponen de alojamiento en 2017

\begin{tabular}{|l|c|c|c|r|r|r|r|}
\hline $\begin{array}{l}\text { Población de } \\
\text { municipios }\end{array}$ & Casos & $\begin{array}{c}\text { Plazas } \\
\mathbf{2 0 0 4}\end{array}$ & $\begin{array}{c}\text { Plazas } \\
\mathbf{2 0 1 7}\end{array}$ & $\begin{array}{c}\text { Población } \\
\mathbf{2 0 0 4}\end{array}$ & $\begin{array}{c}\text { Población } \\
\mathbf{2 0 1 7}\end{array}$ & $\begin{array}{r}\text { Variación } \\
\text { plazas } \\
\mathbf{2 0 0 4 - 2 0 1 7}\end{array}$ & $\begin{array}{r}\text { Variación } \\
\text { población } \\
\mathbf{2 0 0 4 - 2 0 1 7}\end{array}$ \\
\hline Menos de 500 & 74 & 717 & 2.619 & 25.855 & 22.285 & $\begin{array}{r}1.902 \\
(265,3 \%)\end{array}$ & $\begin{array}{r}-3.570 \\
(-13,8 \%)\end{array}$ \\
\hline De 500 a 1.000 & 60 & 2.852 & 5.405 & 49.058 & 43.697 & $\begin{array}{r}2.553 \\
(89,5 \%)\end{array}$ & $\begin{array}{r}-5.361 \\
(-10,9 \%)\end{array}$ \\
\hline De 1.001 a 2.000 & 67 & 4.881 & 6.981 & 108.117 & 99.131 & $\begin{array}{r}2.100 \\
(43,0 \%)\end{array}$ & $\begin{array}{r}-8.986 \\
(-8,3 \%)\end{array}$ \\
\hline De 2.001 a 5.000 & 53 & 7.718 & 10.533 & 169.094 & 163.947 & $\begin{array}{r}2.815 \\
(36,5 \%)\end{array}$ & $\begin{array}{r}-5.147 \\
(-3,0 \%)\end{array}$ \\
\hline De 5.001 a 10.000 & 23 & 2.784 & 3.981 & 159.513 & 152.672 & $\begin{array}{r}1.197 \\
(43,0 \%)\end{array}$ & $\begin{array}{r}-6.841 \\
(-4,3 \%)\end{array}$ \\
\hline $\begin{array}{l}\text { De 10.001 a } \\
20.000\end{array}$ & 6 & 1.425 & 1.832 & 84.556 & 87.918 & $\begin{array}{r}407 \\
(28,6 \%)\end{array}$ & $\begin{array}{r}3.362 \\
(4,0 \%)\end{array}$ \\
\hline $\begin{array}{l}\text { De 20.001 a } \\
50.000\end{array}$ & 4 & 2.101 & 2.505 & 125.221 & 138.173 & $\begin{array}{r}404 \\
(19,2 \%)\end{array}$ & $\begin{array}{r}12.952 \\
(10,3 \%)\end{array}$ \\
\hline Más de 50.000 & 3 & 5.492 & 7.433 & 279.580 & 304.934 & $\begin{array}{r}1.941 \\
(35,3 \%)\end{array}$ & $\begin{array}{r}25.354 \\
(9,1 \%)\end{array}$ \\
\hline
\end{tabular}


El análisis global de los municipios que disponen de algún tipo de alojamiento turístico en 2017, refleja que cuando su población es inferior a 10.000 habitantes continúan perdiendo residentes, alcanzando porcentajes especialmente destacados cuando se trata de aquellos que tienen 2.000 habitantes o menos. Dicha circunstancia contrasta con el hecho de que constituyen el grupo donde el incremento de plazas de alojamiento ha sido más significativo. En cambio, el resto de grupos cuya población supera los 10.000 habitantes, continúa con su modelo de crecimiento demográfico, a la vez que también lo hace en el volumen de plazas.

Contrariamente a lo que se podría suponer, la situación que caracteriza al conjunto de municipios que carecen de alojamiento en 2017 refleja cierto contraste, ya que no existen municipios carentes de alojamiento cuando su población supera los 10.000 habitantes, y los municipios con población inferior a 2.000 habitantes continúan perdiendo población. Sin embargo, existen casos cuya población está comprendida entre los 2.001 y los 10.000 habitantes en los que se ha producido un aumento de población en el periodo analizado.

Cuadro $N^{\circ} 4$

Población y plazas de hospedaje en municipios que no disponen de alojamiento en 2017

\begin{tabular}{|l|c|r|r|r|}
\hline Población de municipios & Casos & \multicolumn{1}{c|}{$\begin{array}{c}\text { Población } \\
\mathbf{2 0 0 4}\end{array}$} & \multicolumn{1}{c|}{$\begin{array}{c}\text { Población } \\
\mathbf{2 0 1 7}\end{array}$} & $\begin{array}{c}\text { Variación población } \\
\text { 2004-2017 }\end{array}$ \\
\hline Menos de 500 & 41 & 12.605 & 10.915 & $-1.690(-13,4 \%)$ \\
\hline De 500 a 1.000 & 35 & 24.310 & 25.552 & $1.242(5,1 \%)$ \\
\hline De 1.001 a 2.000 & 16 & 21.478 & 20.205 & $-1.273(-5,9 \%)$ \\
\hline De 2.001 a 5.000 & 5 & 9.784 & 12.094 & $2.310(23,6 \%)$ \\
\hline De 5.001 a 10.000 & 1 & 6.115 & 6.255 & $140(2,3 \%)$ \\
\hline Más de 10.000 & 0 & 0 & 0 & 0 \\
\hline
\end{tabular}

Fuente: Elaboración propia a partir de INE y Junta de Extremadura

El análisis territorial refleja con excelente detalle el comportamiento dispar que se ha descrito anteriormente, pues la evolución de la población es regresiva en la mayor parte de los municipios; mientras, el aumento de la planta de alojamientos y su capacidad afecta a buena parte de ellos.

Obviamente, los núcleos donde se registra mayor incremento demográfico se corresponden con los más poblados, correspondiéndose también con los que computan un particular aumento en la oferta de alojamiento. A pesar de ello, no se puede obviar que se producen ciertas alteraciones en municipios pequeños, tanto en una variable como en otra, coincidiendo con las áreas que tienen mayor desarrollo turístico, pero también económico, merced al desarrollo agrario alcanzado.

El tratamiento estadístico individualizado entre las 3 tipologías de alojamiento existentes (hotelero, extrahotelero y rural) y la población, tanto de 2004 como de 2017, llevado a cabo mediante una matriz de correlación lineal, muestra que, en líneas generales, se mantienen las tendencias en sendos años, con comportamientos que apenas varían los coeficientes obtenidos. De este modo se observa que la oferta vinculada al alojamiento hotelero tiene especial predilección por los municipios de mayor entidad demográfica. Ello se ve reflejado por un coeficiente de correlación de 
Figura No 2

Evolución de la población y de la oferta de alojamiento

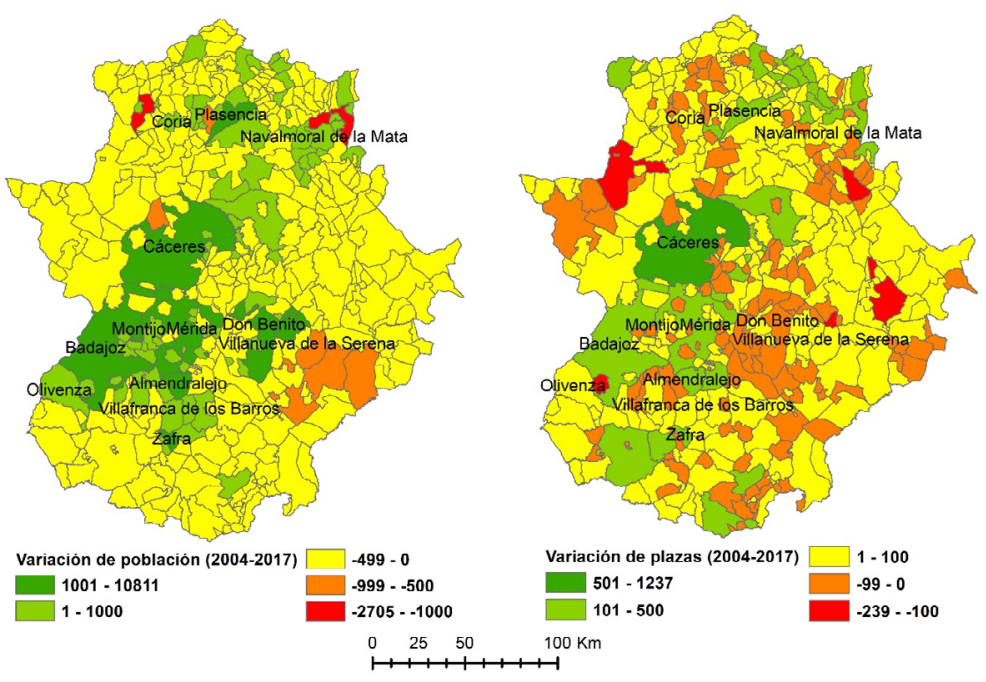

Fuente: Elaboración propia

0,871 y 0,878, calculados para 2004 y 2017 respectivamente. En cambio, la situación que muestra el alojamiento extrahotelero es bien diferente, puesto que los coeficientes, pese a ser significativos a nivel 0,01, tan sólo alcanzan 0,182 y 0,252. Mientras tanto, la correlación obtenida para los alojamientos rurales y la población en 2004 y 2017, no es revelador, pues sus índices son de 0,005 y de -0,026. De este análisis estadístico se deduce que no existe aparente relación entre la capacidad de hospedaje y la población en una tipología de alojamiento concreta, la rural. En cambio, sí existe en las otras dos variedades de hospedaje, siendo esta muy marcada en el caso de la hotelera.

Cuadro No 5

Matriz de correlación lineal

\begin{tabular}{|c|c|c|c|c|c|c|c|c|}
\hline & РНOT04 & PHOT17 & PEHOTO4 & PEHOT17 & PRURO4 & PRUR17 & РOBO4 & POB17 \\
\hline PHOTO4 & 1 &, $982^{* \star}$ &, $339^{* *}$ & $428^{* \star}$ & ,080 & ,081 & $871^{* *}$ & $875^{\star *}$ \\
\hline PHOT17 & $982^{* \star}$ & 1 & $348^{* \star}$ & $446^{* \star}$ & ,070 & 075 & $875^{* \star}$ & $878^{* *}$ \\
\hline PEHOTO4 &, $339^{* *}$ &, $348^{* *}$ & 1 &, $938^{* *}$ &, $428^{* *}$ &, $562^{* *}$ &, $182^{* *}$ & , 182* \\
\hline PEHOT17 &, $428^{* *}$ & $.446^{* *}$ & $938^{* *}$ & 1 & $411^{* *}$ &, $539^{* *}$ &, $252^{* *}$ & ,252* \\
\hline PRURO4 &, 080 & , 070 & $428^{* *}$ &, $411^{* \star}$ & 1 & ,728** &,- 005 &,- 007 \\
\hline PRUR17 & 081 & .075 &, $562^{* *}$ &, $539^{* *}$ &, $728^{* *}$ & 1 &,- 022 &,- 026 \\
\hline POBO4 & ,871** & $875^{\star *}$ &, $182^{* *}$ & $252^{* *}$ &,- 005 &,- 022 & 1 & $999^{* *}$ \\
\hline POB17 & $875^{* *}$ & $878^{* *}$ & $182^{* *}$ &, $252^{* *}$ &,- 007 &,- 026 & $999^{* \star}$ & 1 \\
\hline
\end{tabular}

** La correlación es significativa al nivel 0,01 (bilateral).

* La correlación es significante al nivel 0,05 (bilateral).

PHOTO4: Plazas en alojamiento hotelero en 2004. PHOT17: Plazas en alojamiento hotelero en 2017. PEHOTO4: Plazas en alojamiento extrahotelero en 2004. PEHOT17: Plazas en alojamiento extrahotelero en 2017. PRURO4: Plazas en alojamiento rural en 2004. PRUR17: Plazas en alojamiento rural en 2017. POBO4: Población en 2004. POB17: Población en 2017

Fuente: Elaboración propia 
El análisis territorial refleja situaciones variadas según la evolución experimentada por la población y la capacidad de hospedaje entre 2004 y 2017. Si se considera la oferta conjunta de alojamientos, se observa que hay 83 municipios que tienen crecimiento demográfico. De ellos, 5 disminuyen el conjunto de plazas ofertadas; 4 presentan una oferta similar en los años utilizados para ilustrar el cambio; 21 carecen de plazas y en los restantes 53 municipios se produce un aumento de las mismas.

Cuadro No 6

Síntesis de comportamiento demográfico y de hospedaje

\begin{tabular}{|c|c|c|c|c|c|c|}
\hline & Tipo & Municipios & $\begin{array}{c}\text { Población } \\
2004 \\
\end{array}$ & \begin{tabular}{|c|} 
Población \\
2017 \\
\end{tabular} & $\begin{array}{c}\text { Plazas } \\
2004 \\
\end{array}$ & \begin{tabular}{|c|c|} 
Plazas \\
2017 \\
\end{tabular} \\
\hline \multirow{8}{*}{ 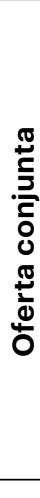 } & $\begin{array}{l}\text { Aumenta población y disminuyen } \\
\text { plazas }\end{array}$ & 5 & 55.769 & 60.700 & 1.111 & 817 \\
\hline & Aumenta población y no tiene plazas & 21 & 20.949 & 27.734 & 0 & 0 \\
\hline & Aumenta población y plazas similares & 4 & 4.866 & 5.256 & 126 & 126 \\
\hline & Aumentan población y plazas & 53 & 549.773 & 591.874 & 13.332 & 19.030 \\
\hline & $\begin{array}{l}\text { Disminuye población y aumentan } \\
\text { plazas }\end{array}$ & 201 & 334.738 & 303.813 & 11.010 & 19.957 \\
\hline & Disminuye población y no tiene plazas & 78 & 53.954 & 47.790 & 9 & 6 \\
\hline & Disminuye población y plazas similares & 15 & 32.928 & 30.204 & 285 & 285 \\
\hline & Disminuyen población y plazas & 11 & 22.309 & 20.407 & 2.097 & 1.068 \\
\hline & TOTAL & 388 & 1.075.286 & 1.087.778 & 27.970 & 41.289 \\
\hline \multirow{9}{*}{ 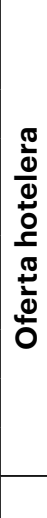 } & $\begin{array}{l}\text { Aumenta población y disminuyen } \\
\text { plazas }\end{array}$ & 7 & 62.027 & 67.204 & 977 & 825 \\
\hline & Aumenta población y no tiene plazas & 36 & 33.272 & 40.738 & 0 & 0 \\
\hline & Aumenta población y plazas similares & 9 & 17.290 & 18.478 & 430 & 430 \\
\hline & Aumentan población y plazas & 31 & 518.768 & 559.144 & 8.888 & 11.876 \\
\hline & $\begin{array}{l}\text { Disminuye población y aumentan } \\
\text { plazas }\end{array}$ & 54 & 150.429 & 137.192 & 2.208 & 3.898 \\
\hline & Disminuye población y no tiene plazas & 191 & 153.706 & 136.289 & 0 & 0 \\
\hline & Disminuye población y plazas similares & 36 & 72.978 & 67.056 & 937 & 937 \\
\hline & Disminuyen población y plazas & 24 & 66.816 & 61.677 & 1.342 & 1.112 \\
\hline & TOTAL & 388 & 1.075 .286 & 1.087.778 & 14.782 & 19.078 \\
\hline \multirow{9}{*}{ 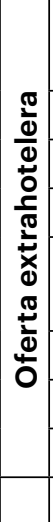 } & $\begin{array}{l}\text { Aumenta población y disminuyen } \\
\text { plazas }\end{array}$ & 1 & 3.837 & 4.202 & 138 & 0 \\
\hline & Aumenta población y no tiene plazas & 61 & 325.252 & 352.247 & 0 & 0 \\
\hline & Aumenta población y plazas similares & 2 & 11.424 & 11.717 & 315 & 315 \\
\hline & Aumentan población y plazas & 19 & 290.844 & 317.398 & 3.206 & 4.395 \\
\hline & $\begin{array}{l}\text { Disminuye población y aumentan } \\
\text { plazas }\end{array}$ & 55 & 124.777 & 112.843 & 4.151 & 6.229 \\
\hline & Disminuye población y no tiene plazas & 242 & 298.201 & 269.943 & 0 & $\underline{0}$ \\
\hline & Disminuye población y plazas similares & 3 & 5.107 & 4.845 & 1.261 & 1.261 \\
\hline & Disminuyen población y plazas & 5 & 15.844 & 14.583 & 1.424 & 96 \\
\hline & TOTAL & 388 & 1.075.286 & 1.087 .778 & 10.495 & 12.296 \\
\hline
\end{tabular}




\begin{tabular}{|c|c|c|c|c|c|c|}
\hline & Tipo & Municipios & \begin{tabular}{|c|} 
Población \\
2004
\end{tabular} & $\begin{array}{c}\text { Población } \\
2017\end{array}$ & $\begin{array}{c}\text { Plazas } \\
2004 \\
\end{array}$ & $\begin{array}{c}\text { Plazas } \\
2017\end{array}$ \\
\hline \multirow{9}{*}{$\begin{array}{l}\bar{\sigma} \\
\frac{\pi}{2} \\
\frac{\pi}{2} \\
\frac{0}{0} \\
\text { to }\end{array}$} & $\begin{array}{l}\text { Aumenta población y disminuyen } \\
\text { plazas }\end{array}$ & 4 & 75.574 & 84.390 & 114 & 28 \\
\hline & Aumenta población y no tiene plazas & 46 & 345.106 & 377.294 & 0 & 0 \\
\hline & Aumenta población y plazas similares & 0 & 0 & 0 & 0 & 0 \\
\hline & Aumentan población y plazas & 33 & 210.677 & 223.880 & 501 & 2.104 \\
\hline & $\begin{array}{l}\begin{array}{l}\text { Disminuye población y aumentan } \\
\text { plazas }\end{array} \\
\end{array}$ & 187 & 297.431 & 272.848 & 1.704 & 7.568 \\
\hline & Disminuyen población y no tiene plazas & 102 & 118.287 & 103.747 & 0 & 0 \\
\hline & Disminuyen población y plazas & 11 & 16.984 & 15.581 & 262 & 103 \\
\hline & $\begin{array}{l}\text { Disminuyen población y plazas } \\
\text { similares }\end{array}$ & 5 & 11.227 & 10.038 & 112 & 112 \\
\hline & TOTAL & 388 & 1.075 .286 & 1.087.778 & 2.693 & 9.915 \\
\hline
\end{tabular}

Fuente: Elaboración propia

La situación que presentan los municipios donde disminuye la población, 305 en total, muestra que en la mayor parte de ellos se produce un aumento significativo en el volumen de hospedaje ofrecido por todos los tipos de alojamiento. En concreto, 201 municipios evidencian un aumento de capacidad, mientras que 78 carecen de cualquier tipo de oferta; manteniéndose el mismo número de plazas en 15 municipios y, por último, disminuye en 11. Pese a todo, esta situación presenta diferencias en función del tipo de alojamiento analizado, en línea con los resultados obtenidos por la matriz de correlación lineal.

En este sentido, cabe destacar que cuando se analiza el comportamiento de la oferta hotelera definida por el artículo 5 del Decreto 235/2012, de 4 de diciembre, por el que se establece la ordenación y clasificación de los Alojamientos Turísticos Hoteleros de la Comunidad Autónoma de Extremadura (DOE n 236), tan sólo aumenta la población en 83 de los 388 municipios analizados. Este aumento de población se produce en núcleos que carecen de plazas en esta modalidad de alojamiento (36 municipios); en otros que presentan un volumen similar de oferta en los años considerados (9 municipios); e incluso en lugares donde disminuye el número de plazas ofertadas (7 municipios), circunstancia que afecta también a localidades con un volumen de población importante, si bien, el descenso de plazas de alojamiento hotelero es poco significativo. Sin embargo, 31 municipios aumentan, además de la población, la capacidad de hospedaje. Normalmente se corresponde con los núcleos de mayor entidad, aunque también se observa dicho comportamiento en áreas que se localizan próximas a ellos y, en algunos casos, se hallan en las proximidades de las principales vías de comunicación y de zonas turísticas consolidadas.

El resto de municipios presenta un claro descenso demográfico, siendo la tónica predominante que carezcan de plazas de alojamiento hotelero, hecho que afecta a 191 municipios, casi todos con una población inferior a 2.000 habitantes. Por otra parte, en otros 36 , también de pequeño tamaño, se mantiene un volumen similar de plazas en los años considerados; mientras, en 24, además de perder población, disminuyen las plazas en alojamiento hotelero. Asimismo, es destacable que, incluso perdiendo población, 55 municipios aumentan las plazas hoteleras, de forma variable. 
La oferta extrahotelera presenta características peculiares, al igual que los dos tipos básicos de alojamientos que la componen, el campamento y los apartamentos turísticos, regulados por los artículos 63 y 61 de la Ley 2/2011 de desarrollo y modernización del turismo. El análisis de la misma refleja que de los 83 municipios que ganan población entre 2004 y 2017, únicamente uno pierde capacidad de hospedaje; dos mantienen el mismo número de plazas; mientras que 61 carecen de este tipo de alojamiento. Como contraste, 19 municipios aumentan de forma considerable su volumen de plazas extrahoteleras, destacando entre ellos los principales núcleos turísticos de Extremadura.

Por otra parte, del nutrido grupo de municipios que registra un descenso demográfico, 242 carecen de esta variedad de alojamiento; 3 mantienen el mismo volumen de plazas en los años de referencia y en otros 5 disminuye también la capacidad de hospedaje en este tipo de establecimiento. No obstante, hay que considerar que, pese a la disminución demográfica, en 55 municipios se registra un aumento variable en la capacidad en este tipo de planta.

La evolución combinada de la población con la capacidad de hospedaje que ofertan los alojamientos rurales muestra que, de los 83 municipios con crecimiento demográfico, la mayoría (46) carece de plazas en esta modalidad de acomodo, y disponen de un volumen de residentes muy variable como lo certifica el municipio de Badajoz y el de Aldehuela de Jerte, con casi 150.000 y menos de 400 habitantes, respectivamente. En cambio, en 33 municipios se incrementan las plazas de alojamiento rural, pese a contar con una población censada, asimismo variable. Sin embargo, 4 municipios pierden plazas en esta modalidad de alojamiento. Uno de ellos se aproxima a los 20.000 habitantes y el otro supera los 50.000; mientras que los otros se encuentran en el intervalo de 2.000 a 10.000 residentes.

La situación que caracteriza a los municipios que pierden población desvela que una gran mayoría de los mismos ha aumentado el volumen de plazas en alojamientos rurales, demostrando la apuesta inequívoca por el desarrollo de la tipología turística rural en buena parte de Extremadura. De hecho, 187 municipios aumentan el volumen de plazas ofertadas. Frente a ellos, 102 carecen de este tipo de oferta; 5 mantienen el mismo volumen de hospedaje y en 11 municipios disminuyen las plazas ofertadas.

Los resultados obtenidos reflejan con claridad que la implantación de alojamientos no es un elemento decisivo en el contexto territorial analizado para frenar la pérdida de población, pues, se observa que los núcleos de menor entidad demográfica continúan perdiendo efectivos de forma generalizada. De hecho, de los 375 municipios que en 2017 disponían de menos de 10.000 habitantes, 305 pierden un total de 41.715 residentes; por el contrario, 70 ganan 12.539 .

Este dispar comportamiento demográfico se produce a pesar de que 243 de ellos ven como se incrementa su capacidad de hospedaje en 11.868 plazas, de las que 2.921 se corresponden con aquellos municipios que ganan población y 8.947 con los que la pierden. Aun así, no se debe obviar que en 16 municipios se pierden plazas de alojamiento, pues, aunque 4 de ellos ganen población, pierden 208 plazas y 12 pierden 1.032, si bien ellos también descienden en población.

Si esta es la situación que se da en los entornos rurales, la situación se torna bastante más simple cuando se compara con la que caracteriza a los municipios que superan los 10.000 residentes. En ellos, la población aumenta, al igual que lo hace la planta de alojamientos. 
Figura No 3

Evolución de la población y de la oferta de alojamiento (2004-2017)
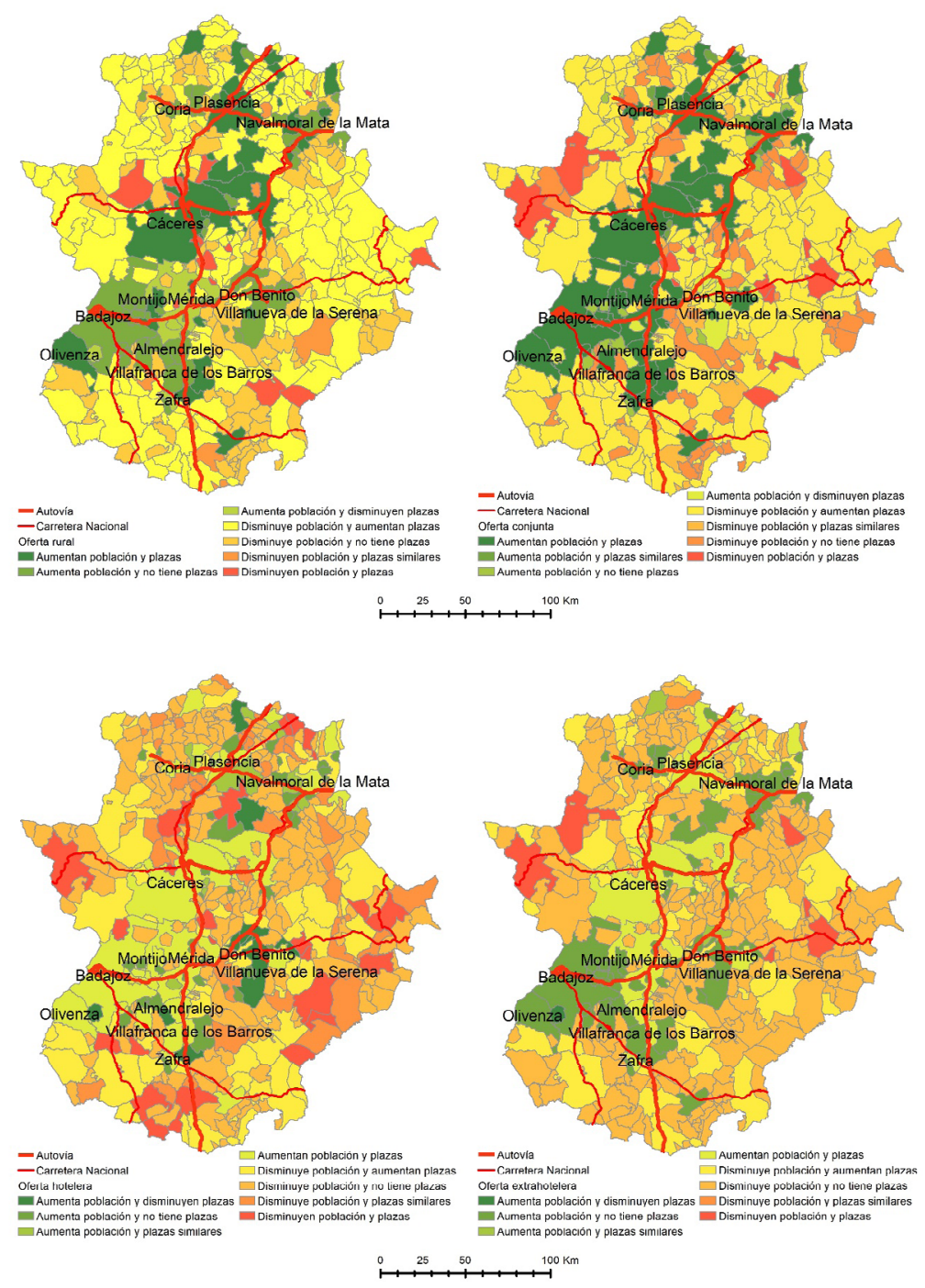

Fuente: Elaboración propia

El contraste entre el mundo rural y el urbano es patente por el comportamiento desigual, si bien resulta significativo que la apuesta realizada por la implantación de una abundante oferta de alojamientos rurales no ha sido capaz de cumplir de forma generalizada con uno de los objetivos básicos que se plantean las políticas europeas de desarrollo rural, fijar la población e impedir la paulatina despoblación de los municipios rurales.

Las causas que impiden la recuperación demográfica de muchos núcleos rurales, a pesar de la masiva implantación de alojamientos en ellos, son múltiples, aunque a nuestro juicio las vinculadas al desarrollo turístico pueden sintetizarse en dos muy concretas. 
La primera consiste en que la elección de lugares para la localización de alojamientos no siempre se corresponde con la ubicación de los atractivos más demandados por los turistas rurales, profusamente analizados en el entorno extremeño. Entre ellos destacan la presencia de relieves montañosos, de cursos de agua cristalinos y de zonas adaptadas para el baño, como las piscinas naturales (Sánchez, Sánchez \& Rengifo, 2017). Ello, obviamente se traduce en una escasa competitividad, sobre todo durante la época estival, que concentra buena parte de las pernoctaciones (Sánchez \& Rengifo, 2018).

La segunda radica en que el desarrollo turístico no debe centrarse exclusivamente en la puesta en servicio del alojamiento, sino que debe ir acompañada de unos servicios complementarios de calidad, donde la oferta de actividades debe orientarse específicamente a las fortalezas de cada territorio. Esto puede beneficiar a muchos alojamientos establecidos en las áreas menos demandadas por los turistas, donde es muy fácil encontrar recursos paisajísticos de primer nivel que facilitarían la puesta en marcha de productos turísticos diferenciados. En este sentido cabe señalar que prácticamente el $33 \%$ del territorio extremeño se encuentra preservado bajo alguna figura de protección ambiental integrada en la Red ENP, Red Natura 2000 o bien de otras figuras de protección de espacios. Por todo ello, pensamos que si realmente se especula con que el turismo puede servir de base para iniciar el desarrollo socioeconómico generalizado del mundo rural y, a la postre, contribuir a fijar la población a dichos entornos, es preciso acometer políticas turísticas conducentes a garantizar el diseño de productos turísticos adaptados al entorno inmediato, favoreciendo la implantación de actividades que contribuyan a hacer más atractivo el espacio y, sobre todo, apostar por las tendencias que marcan la demanda actual del turismo rural: naturaleza, ecología, etnografía, gastronomía y un largo etcétera (Sánchez, Rengifo \& Martín, 2018; Sánchez, Rengifo \& Blas, 2019).

\section{Conclusiones}

Tras la realización de este estudio extraemos las siguientes conclusiones:

La primera y una de las más importantes muestra que Extremadura está inmersa en un claro proceso de regresión demográfica, ya que existe una clara pérdida de población desde la década de los sesenta. En la actualidad, tan sólo reside en la comunidad el $79 \%$ de la población que lo hacía en 1960, situación que contrasta enormemente con la que se produce en el conjunto de España, donde la población ha aumentado un $66 \%$ durante ese mismo periodo. Además, la pérdida demográfica se agrava aún más con el paulatino envejecimiento de la población y, sobre todo, porque afecta con mayor virulencia a los núcleos rurales que tienen menos de 5.000 habitantes. A pesar de todo, el acusado descenso demográfico aparece enmascarado durante los años 2004 y 2017, donde se observa un ligero incremento de población debido a la crisis económica y, consecuentemente, a la pérdida de poder de atracción de las ciudades más dinámicas del país.

La segunda es que la oferta de alojamientos ha seguido una política claramente expansiva, ya que se ha pasado de ofrecer 27.970 plazas en 2004 a 41.289 en 2017, lo que supone un incremento del $47,6 \%$. El aumento de la capacidad de alojamiento ha afectado a todas las tipologías, aunque en distinto grado. Así, es destacable que las plazas en alojamientos hoteleros hayan crecido el $29,1 \%$; 
las ofertadas por los alojamientos extrahoteleros lo han hecho en un $17,2 \%$; y, por último, las ofrecidas por la modalidad de establecimientos rurales han subido un $368 \%$. Ello pone de manifiesto la gran apuesta que se ha realizado por desarrollar el turismo en todos los espacios y, en concreto, se ha apostado por la modalidad del alojamiento rural como medida para contribuir al desarrollo socioeconómico de estos espacios, en línea con lo promovido por las iniciativas europeas.

La tercera conclusión que se obtiene es que no hay evidencias de que el desarrollo de la planta de alojamientos turísticos y de su capacidad de hospedaje tenga una relación directa con el aumento o la disminución de la población, ya que este proceso de regresión demográfica afecta a muchos municipios (305 de los 388), independientemente de su capacidad de alojamiento. Lejos de ello, se constata que, en buena parte de los municipios, sobre todo en los de menor tamaño, continúa el proceso de pérdida de población, aunque el volumen de plazas aumente de forma significativa. Se pone así de manifiesto que las políticas seguidas no han conseguido de forma generalizada contribuir a la fijación de la población, si bien, lo han hecho de forma muy puntual.

La cuarta y última conclusión refleja que el aumento de la población se produce en los núcleos de mayor entidad demográfica y en los núcleos eminentemente rurales que reúnen las mejores facilidades turísticas, puestas de manifiesto mediante la presencia de recursos turísticos de notable valía.

\section{Agradecimientos}

La presente publicación forma parte del proyecto de investigación "Diseño y elaboración de productos estratégicos diferenciados para la potenciación del turismo rural en Extremadura. De la detección de problemas a la propuesta de soluciones basadas en criterios geoestadísticos", cuya clave es IB 16040, y se encuentra financiado por la Consejería de Economía e Infraestructuras de la Junta de Extremadura y por el Fondo Europeo de Desarrollo Regional (FEDER).

\section{Referencias bibliográficas}

Ley 45/2007 para el desarrollo sostenible del medio rural. Boletín Oficial del Estado, Madrid, España, 14 de diciembre de 2007.

Real Decreto 1336/2011, de 3 de octubre, por el que se regula el contrato territorial como instrumento para promover el desarrollo sostenible del medio rural. Boletín Oficial del Estado, Madrid, España, 4 de octubre de 2011.

ATANCE, I.; GARCÍA CORRAL, A.; MARTÍNEZ JÁVEGA, M.P. \& URRUELA, J. La población rural en España: un enfoque a escala municipal. Economía Agraria y Recursos Naturales, 2010, № 10 (1), p. 35-57.

BARAJA, E. La despoblación, un fenómeno demográfico todavía vigente en Castilla y León. En F. GARCÍA PASCUAL, F. (Coord.). La lucha contra la despoblación todavía necesaria. Políticas y estrategias sobre la despoblación rural en el siglo XXI. Zaragoza: CEDDAR, 2003, p. 105-126. 
BARKE, M. Rural tourism in Spain. International Journal of Tourism Research, 2004, No 6 (3), p. $137-$ 149. doi:10.1002/jtr.480

BRINGAS, J. M. El despoblamiento rural: causas y consecuencias. Arquitectura: Revista del Colegio Oficial de Arquitectos de Madrid (COAM), 1963, № 53, p. 53-56.

BUSTOS, Ma L. (2018). Evolución de la población rural en la provincia de Salamanca. Boletín de la Asociación de Geógrafos Españoles, 2018, № 77, p. 200-228. doi: 10.21138/bage.2539

CAMARERO, L. (Coord). La población rural de España. De los desequilibrios a la sostenibilidad social. Colección Estudios Sociales, 2009, № 27. p. 41. Fundación "la Caixa": Barcelona. Disponible en: http://www.academia.edu/download/39253010/Ofcfd507e71f5a505e000000.pdf

CAMARERO, L.; SAMPEDRO, R. ¿Por qué se van las mujeres? El continuum de movilidad como hipótesis explicativa de la masculinización rural. Revista Española de Investigaciones Sociológicas (REIS), 2008, No 1, p. 73-105. Disponible en: https://www.ingentaconnect.com/content/cis/ reis/2008/00000124/00000001/art00003\#

CÁNOVES, G.; VILLARINO, M.; PRIESTLEY, G.; BLANCO, A. Rural tourism in Spain: an analysis of recent evolution. Geoforum, 2004, No 35 (6), p. 755-769.

CÀNOVES, G., VILLARINO, M., \& HERRERA, L. Políticas públicas, turismo rural y sostenibilidad: difícil equilibrio. Boletín de La Asociación de Geógrafos Españoles, 2006, № 41, p. 199-217. Disponible en: https://www.age-geografia.es/ojs/index.php/bage/article/view/1997/0

ENTRENA-DURÁN, F. La ruralidad en España: de la mitificación conservadora al neorruralismo. Cuadernos de desarrollo rural, 2012, No 69, p. 39-65.

ERDOZÁIN, P.; MIKALERENA, F. Algunas consideraciones acerca de la evolución de la población rural en España en el siglo XIX. Historia agraria: Revista de agricultura e historia rural, 1996, № 12, p. 91-118.

ESCRIBANO, J. El valor de los servicios educativos y sanitarios en los procesos de atracción y fijación de población en medio rural. Ager, Revista de estudios sobre despoblación y desarrollo rural, 2012, No 13, p. 11-51. doi: 10.4422/ager.2011.07

ESCRIBANO, J. Servicios educativos y sanitarios elementales en el medio rural: percepción social e influencia sobre la calidad de vida. Estudios Geográficos, 2012, № 272, p. 35-61. doi: 10.3989/ estgeogr.201202

GARCÍA, J.L. El turismo rural como factor diversificador de rentas en la tradicional economía agraria. Estudios Turísticos, 1996, № 132, p. 47-61. 
GARCÍA, B.. Del agrarismo a la terciarización: modelos de actividad en la sociedad rural. En GONZÁLEZ, J.J. y GÓMEZ, C. (Coords). Agricultura y sociedad en la España contemporánea. Madrid: CIS, Ministerio de Agricultura, Pesca y Alimentación. 1997, p. 1020.

GURRÍA, J.L.; SÁNCHEZ, J.M. \& HERNÁNDEZ, A.Ma . Movilidad y desarrollo rural. En GURRÍA GASCÓN, J.L. (Ed.), Políticas europeas y nuevas dinámicas rurales en Extremadura (1991-2010). Cáceres: Servicio de Publicaciones de la Universidad de Extremadura, 2017, p. 77-150. Disponible en: http:// dehesa.unex.es/bitstream/handle/10662/5240/978-84-617-8692-3. pdf?sequence=1\&isAllowed=y

JURADO, J.M. \& PAZOS, F. Población y turismo rural en territorios de baja densidad demográfica en España. Boletín de la Asociación de Geógrafos Españoles, 2016, № 71, p. 247-272. doi: 10.21138/ bage.2282

LÓPEZ, L.; ABELLÁN, A. \& GODENAU, D. Despoblación, envejecimiento y territorio: un análisis sobre la población española. León: Universidad de León, 2009

MARTÍN, F. \& MARTíN, I. Los espacios rurales españoles: ¿territorios donde se produce y consume turismo o destinos sostenibles en entornos competitivos? El caso del Nordeste. Boletín de la Asociación de Geógrafos Españoles, 2014, № 64, p. 201-226.

MARTíN, I. \& MARTíN, F. Interpretación del patrimonio y gestión sostenible del turismo en espacios rurales. Los casos de Montejo de la Sierra y Patones. Boletín de la Asociación de Geógrafos Españoles, 2016, № 72, p. 169-193. doi: 10.21138/bage.2336

MESA, M. (2000). Desarrollo Local y Turismo: El Turismo Rural. En PÉREZ RAMíREZ, B. y CARRILLO BENITO, E. (Ed.), Desarrollo Local: Manual de Uso. Madrid: ESIC., 2000, p. 555-600.

MORENO, D.; RUÍZ, V. \& ESPÍN, D. Turismo y desarrollo rural en la comarca de las Tieras Altas de Lorca (Región de Murcia, España). Caso de estudio: El Proyecto "Cinturón Espartaria". Cuadernos de Turismo, 2017, № 36, p. 111-119.

PANIAGUA, A. Rurality, identity and morality in remote rural areas in northern Spain. Journal of Rural Studies, 2017, No 35, p. 49-58. doi: https://doi.org/10.1016/j.jrurstud.2014.03.009

POZO, E.; GARCÍA, J. La revitalización demográfica de las comarcas rurales madrileñas. En LÓPEZ, L.; ABELLÁN, A.; GODENAU, D. (Coord). Despoblación, envejecimiento y territorio: un análisis sobre la población española. León: Universidad de León, 2009, p. 275-288.

PRATS, L. (2011). La viabilidad turística del patrimonio. PASOS. Revista de Turismo y Patrimonio Cultura, No 9(2), p. 249-264. doi: https://doi.org/ISSN 1695-7121

RENGIFO, J.I. \& SÁNCHEZ, J.M. Balance del crecimiento del turismo rural en Extremadura (19922015): dos décadas de éxito y decepciones. $X$ Congreso Internacional de Turismo Rural y DesarroIlo Sostenible. Santiago de Compostela: Universidade Federal do Rio Grando do Sul; Grupo Merca- 
dos Nâo Agrícolas Rurais; Análisis Territorial (USC), Centro de Estudos e Investigacións Turísticas (CETUR), 2016, p. 275-290.

RENGIFO, J.I. \& SÁNCHEZ, J.M. El patrimonio en Extremadura: un mecanismo para la cooperación transfronteriza. Polígonos. Revista de Geografía, 2017, № 29, p. 223-248. doi: http://dx.doi. org/10.18002/pol.v0i29.5207

ROQUER, S. \& BLAY, J. Del éxodo rural a la inmigración extranjera: el papel de la población extranjera en la recuperación demográfica de las zonas rurales españolas (1996-2006). Scripta Nova, 2008, No 270. Disponible en: http://goo.gl/DEcNSp

SÁNCHEZ, J.M. La influencia de los factores geográficos en el clima de montaña de Extremadura. Hacia una definición, tipificación y delimitación estadístico-sistémica. Fundicot-Extremadura, Universidad de Extremadura, Junta de Extremadura, 1995, p. 228

SÁNCHEZ, J.M.; RENGIFO, J.I. Los espacios naturales protegidos y su capacidad de atracción turística: Referencias al Parque Nacional de Monfragüe (Extremadura-España). En: Intellectual Capital and Regional Develpment: New Landscapes and Challenges for Planning the Space. Coimbra: APDR; Universidade da Beira Interior; RSAl, ersa, 2017, p. 1196-1206.

SÁNCHEZ, J.M.; RENGIFO, J.I. Evolución del sector turístico en la Extremadura del siglo XXI: auge, crisis y recuperación. Lurralde: investigación y espacio, 2019, № 42, p. 19-50. Disponible en: http:// www.ingeba.org/lurralde/lurranet/lur42/42sanchez.pdf

SÁNCHEZ, J.M.; RENGIFO, J.I.; BLAS, R. Geotourist Profile Identification Using Binary Logit Modeling: Application to the Villuercas-Ibores-Jara Geopark (Spain). International Journal of Geo-Information, 2019, No 8, p. 176. doi:10.3390/ijgi8040176

SÁNCHEZ, J.M.; RENGIFO, J.I. \& MARTÍN, L.M. Tourist mobility at the destination toward protected areas: the case-study of Extremadura. Sustainability, 2018, No 10(12), p. 4853. doi:10.3390/ su10124853

SÁNCHEZ, J.M.; SÁNCHEZ, M. \& RENGIFO, J.I. Análisis del equilibrio entre el potencial turístico y la oferta de alojamientos en turismo rural mediante técnicas de estadística espacial. Una aplicación a la provincia de Cáceres (España). Cuadernos de Turismo, 2017, № 39, p. 547-576.

SÁNCHEZ, B. Evolución de la población de los municipios de Extremadura: Aplicaciones paramétricas y semiparamétricas. (Tesis Doctoral). 2011. Disponible en: https://repositorio.uam.es/bitstream/handle/10486/6857/40109_Sanchez_Reyes_Beatriz.pdf?sequence=1

SHARPLEY, R.; ROBERTS, L. (2004). Rural Tourism - 10 years on. International Journal of Tourism Research, No 6(3), p. 119-124. doi: doi.org/10.1002/jtr.478 
SILVESTRE, J. Las emigraciones interiores en España durante los siglos XIX y XX: una revisión bibliográfica. Ager. Revista de Estudios sobre Despoblación y Desarrollo Rural, 2002, № 2, p. $227-$ 248.

SLOMP, H. J. La despoblación del medio rural español. Groningen (Holanda), 2004. Disponible en: http://www.despoblacionaragon.nl/scriptie_spaans/scriptiee.pdf

TAMAYO, M. Diccionario de la investigación científica. México: Limusa, 1990.

TIRADO BALLESTEROS, J.G.; HERNÁNDEZ HERNÁNDEZ, M. Assessing the Impact of EU Rural Development Programs on Tourism. Tourism Planning and Development, 2017, No 14(2), p. 149-166. doi: https://doi.org/10.1080/21568316.2016.1192059

TIRADO, J.G.; HERNÁNDEZ, M. Promoting Tourism through the EU LEADER Programme: Understanding Local Action Group Governance. European Planning Studies, 2018, № 27(2), p. 1-19. doi: https://doi.org/10.1080/09654313.2018.1547368

VINASCO, M. Marco teórico para la construcción de una propuesta de turismo rural comunitario. Revista de Investigación Agraria y Ambiental, 2017, № 8 (1), p. 95-106.

YUBERO, C.; GARCÍA, M. (2019). El turismo en el medio rural en España desde el enfoque de la transferencia de políticas públicas. Boletín de la Asociación de Geografía Española, № 81, 2766, p. 1-34. doi: http://dx.doi.org/10.21138/bage.2766

ZASADA, I.; WELTIN, M.; REUTTER, M.; VERBURG, P.H.; PIORR, A. EU's rural development policy at the regional level-Are expenditures for natural capital linked with territorial needs? Land Use Policy, 2018, No 77, p. 344-35. doi: https://doi.org/10.1016/j.landusepol.2018.05.053 